\title{
Parteimitgliedschaften im Jahre 2010
}

\author{
Oskar Niedermayer
}

Mit der folgenden Dokumentation wird der seit 2001 jährlich erscheinende systematische Überblick über die Entwicklung, regionale Verteilung und sozialstrukturelle Zusammensetzung der Parteimitgliedschaften fortgeschrieben. Zusätzliche Schaubilder sind über das Internet abrufbar (http://www.polsoz.fu-berlin.de/polwiss/forschung/systeme/empsoz/schriften/Arbeitshefte/ahosz18.pdf) ${ }^{1}$. Es werden nur Daten aus der Mitgliederverwaltung der Parteien verwendet ${ }^{2}$. Die Dokumentation der sozialstrukturellen Zusammensetzung der Parteimitgliedschaften enthält keine Angaben über die berufliche Stellung, weil die hierzu vorhandenen Daten den Beruf zum Zeitpunkt des Parteieintritts ohne spätere Aktualisierung wiedergeben und somit die tatsächliche Berufsstruktur der Parteimitgliedschaften nicht korrekt widerspiegeln.

Im Herbst 2008 wurde auf Anregung der Bundesgeschäftsführer vom Verfasser ein Workshop mit allen für die Mitgliederstatistik verantwortlichen Mitarbeitern der Parteien durchgeführt, auf dem eine Reihe von Verbesserungen der Mitgliederstatistik und ihrer Publikation beschlossen wurde. Daher gibt es seit Ende 2008 eine Altersstatistik der Mitglieder mit einer identischen, sehr feinen Altersgruppeneinteilung sowie eine Statistik der Eintritte, Austritte und Todesfälle und der Eintritte nach Geschlecht und Alter. Zusätzlich werden Daten zur Rekrutierungsfähigkeit der Parteien und zur Über- beziehungsweise Unterrepräsentation bestimmter Bevölkerungsgruppen in den Parteimitgliedschaften publiziert.

Trotz des Bemühens aller Parteien, ihre Mitgliedschaft wenigstens zu stabilisieren, mussten CDU, SPD und CSU im Jahre 2010 wieder größere Mitgliederverluste hinnehmen, wobei es zum zweiten Mal hintereinander die CSU am stärksten traf. Bei der FDP, die 2009 aufgrund des sehr guten Abschneidens bei der Bundestagswahl einen Zuwachs von fast zehn Prozent verbuchen konnte, schlug sich der nachfolgende Absturz in der Wählergunst auch in einem deutlichen Mitgliederrückgang nieder. Für die Grünen brachte der demoskopische Höhenflug 2010 mit zehn Prozent den stärksten Mitgliederzuwachs seit Mitte der 1990er Jahre, während die Linkspartei zum ersten Mal seit der Vereinigung von WASG und PDS 2007 einen Mitgliederrückgang verkraften musste. Betrachtet man den gesamten Zeitraum seit 1990, so haben alle Parteien außer den Grünen Mitglieder verloren, wenn auch in sehr unterschiedlichem Maße. Am stärksten hat es die Linke getroffen, die - trotz des Zuwachses durch die Vereinigung von PDS und WASG - Ende 2010 fast drei Viertel weniger Mitglieder zählt als die PDS Ende 1990. Die Grünen haben durch den starken Zuwachs 2010 - sowohl im Vergleich zu 1990 als auch gemessen an ihrem erst 1998 erreichten Höchststand - Mitglieder hinzugewonnen (vgl. Tabelle 1).

1 Für eine Analyse der Daten seit 1946 vgl. Oskar Niedermayer, Der Wandel des parteipolitischen Engagements der Bürger, in: Steffen Kühnel / ders. / Bettina Westle (Hrsg.), Wähler in Deutschland. Sozialer und politischer Wandel, Gender und Wahlverhalten, Wiesbaden 2009, S. 82 134.

2 Den Geschäftsstellen der Parteien, die dem Verfasser die Daten freundlicherweise zur Verfügung gestellt haben, sei an dieser Stelle ausdrücklich gedankt. 
Betrachtet man statt der absoluten Mitgliederzahlen die Rekrutierungsfähigkeit - also den Anteil der Parteimitglieder an den Parteibeitrittsberechtigten -, dann verändern sich die Größenordnungen zwischen den Parteien, da sie unterschiedliche Mindestalterbestimmungen für den Parteibeitritt haben (ab 14 beziehungsweise 16 Jahren) und die CDU ihre Mitglieder nur außerhalb Bayerns rekrutieren kann, während die CSU auf Bayern beschränkt ist. Die SPD war bis zum Frühjahr 2008 die mitgliederstärkste Partei. Hinsichtlich der Fähigkeit zur Mitgliederrekrutierung wurde sie allerdings schon 1999 von der CDU überholt, wobei beide Parteien wiederum weit hinter der CSU zurückliegen, deren Mitgliedschaft Ende 2009 1,5 Prozent der bayerischen Bevölkerung ab 16 Jahren umfasste. Insgesamt waren 1980, nach dem Hinzukommen der Grünen, in der alten Bundesrepublik fast vier Prozent der beitrittsberechtigten Bevölkerung (knapp zwei Millionen Bürger) in einer der fünf Parteien organisiert. Ende 1989 waren es noch 3,6 Prozent, nach der Vereinigung und dem Dazukommen der damaligen PDS 3,7 Prozent. Ende 2009 gehörten nur noch knapp zwei Prozent der beitrittsberechtigten Bevölkerung einer der sechs wichtigen deutschen Parteien an (für Ende 2010 lagen bei Redaktionsschluss noch keine Bevölkerungsdaten vor). Es ist somit eine kontinuierlich abnehmende gesellschaftliche Verankerung des Parteiensystems zu beobachten, die in neuester Zeit zwar etwas gebremst scheint, aber noch nicht gestoppt ist (vgl. Tabelle 2).

Die beiden großen Parteien mussten im Jahre 2010 Mitgliederverluste in fast allen Bundesländern hinnehmen. Lediglich in Berlin und in Hamburg (nur CDU) konnte leichter Zuwachs erzielt werden. Die FDP, die 2009 ihre Mitgliederzahlen noch flächendeckend das heißt in allen Bundesländern - steigern konnte, verlor 2010 Mitglieder in allen Bundesländern außer dem Saarland. Die Grünen hingegen erzielten in allen Ländern - zum Teil beachtliche - Zugewinne. Die Linkspartei, die 2008 und 2009 in allen westdeutschen Ländern zulegen konnte, verlor 2010 genau dort wieder an Mitgliedern (am drastischsten im Saarland) und setzte zugleich ihren flächendeckenden Mitgliederschwund der letzten Jahre in Ostdeutschland fort (vgl. Tabelle 3).

Bei der Rekrutierungsfähigkeit der Parteien nach Bundesländern zeigte sich auch 2009 das schon seit längerer Zeit bestehende Muster: Bei der SPD und den Grünen bilden die fünf ostdeutschen Bundesländer mit deutlichem Abstand zu allen westdeutschen Ländern das Schlusslicht; bei der CDU weisen nur die Stadtstaaten Berlin, Bremen und Hamburg ähnlich niedrige Rekrutierungsgrade auf wie die ostdeutschen Länder. Bei der FDP bestehen hingegen keine systematischen Ost-West-Unterschiede. Die Linke blieb auch 2009 von ihrer Mitgliederverankerung noch eine ostdeutsche Regionalpartei, die im Westen - mit Ausnahme des Saarlands, der Heimat von Oskar Lafontaine - über eine geringe Organisationsbasis verfügte, aber auch im Osten nirgendwo auch nur annähernd die Rekrutierungsfähigkeit der CDU, SPD und CSU in ihren westdeutschen Hochburgen erreichte (vgl. Tabelle 4).

Beim Anteil der Frauen an den Parteimitgliedschaften ist über einen längeren Zeitraum hinweg kein parteiübergreifender Trend festzustellen. Während der Frauenanteil seit vielen Jahren bei der CDU leicht und bei der SPD und der CSU stärker ansteigt, sinkt er bei den Grünen nach einem zwischenzeitlichen Anstieg 2010 wieder leicht. Bei der FDP setzte sich die seit Mitte der 1990er Jahre kontinuierliche Abnahme des Frauenanteils 2010 zum ersten Mal nicht fort. Die PDS verlor im selben Zeitraum leicht - ein Trend, der sich durch die Vereinigung mit der WASG wesentlich beschleunigte. 2010 ist erstmals wieder ein leichter Anstieg des Frauenanteils zu verzeichnen (vgl. Tabelle 5). 
Der Anteil einer bestimmten Bevölkerungsgruppe unter den Parteimitgliedern sagt noch nichts darüber aus, ob diese Gruppe in den Parteimitgliedschaften gegenüber der Bevölkerung unter- oder überrepräsentiert ist. Um die Art der Repräsentation sozialer Gruppen feststellen und zwischen den Parteien exakt vergleichen zu können, wurden daher so genannte Proportionalitätsquotienten (PQ) gebildet, indem der Anteil einer Gruppe an den Parteimitgliedern durch den Anteil dieser Gruppe an der jeweiligen beitrittsberechtigten Bevölkerung dividiert wurde. PQ-Werte über 1 bedeuten daher eine Überrepräsentation der Gruppe bei den Parteimitgliedern und PQ-Werte unter 1 eine Unterrepräsentation, die umso stärker ist, je kleiner der Wert ist. Die PQ-Werte für den Frauenanteil zeigen, dass die Frauen in den Mitgliedschaften aller Parteien unterrepräsentiert sind, allerdings mit deutlichen Unterschieden: Ende 2009 war zum Beispiel der Anteil der Frauen an der CDUMitgliedschaft (25,5 Prozent) halb so groß wie in der Bevölkerung ab 16 Jahren außerhalb Bayerns (51,4 Prozent), so dass eine Unterrepräsentation vorlag $(\mathrm{PQ}=0,5)$. Am stärksten ist die Unterrepräsentation von Frauen in der CSU, in der PDS war sie bis 2006 am geringsten, die Vereinigung mit der WASG hat sie jedoch ansteigen lassen, und 2008 zog die Linke mit den Grünen gleich (vgl. Tabelle 6).

Betrachtet man die Entwicklung der Altersstruktur der Parteimitgliedschaften in der groben Unterteilung in Jüngere (bis 29/30 Jahre), eine mittlere Kategorie (29/30 bis 59/60 Jahre) und Ältere (ab 60/61 Jahre) bis 2007, so scheint der bis etwa zur Jahrhundertwende zu beobachtende Rückgang des Anteils der jüngeren Parteimitglieder vor allem für die SPD endgültig gestoppt zu sein, denn hier zeigte sich über mehrere Jahre hinweg eine leichte Verbesserung, während die Werte für die CDU und vor allem auch für die FDP in den letzten Jahren vor 2007 wieder abwärts gingen. Den höchsten Anteil an jüngeren Parteimitgliedern wiesen 2007 die Grünen auf, die nach einer Auswertung ihrer Mitgliederdatei erstmals über Daten zur Altersstruktur verfügten. Den geringsten Anteil an Jüngeren und den mit Abstand höchsten Anteil an Älteren verzeichnete bis 2006 die PDS. Durch den Zusammenschluss mit der WASG hat sich die Partei allerdings deutlich „verjüngt“ (vgl. Tabelle 7).

In allen Parteien waren die Jüngeren bis 2007 deutlich unterrepräsentiert und die Älteren überrepräsentiert, wobei sich die Überrepräsentation der Älteren im Zeitverlauf deutlich verstärkt hat. Die Unterrepräsentation der Jüngeren hat hingegen nicht in der Weise zugenommen, wie es die Entwicklung der Anteile der Jüngeren an den Mitgliedschaften vermuten lässt, da der Anteil der Jüngeren an der beitrittsberechtigten Bevölkerung im Zeitverlauf auch abgenommen hat. So waren zum Beispiel 1990 sieben Prozent der CSUMitgliedschaft 16 bis 30 Jahre und 2007 nur noch 5,5 Prozent. Da jedoch der Anteil derselben Altersgruppe an der bayerischen Bevölkerung 1990 28,3 Prozent und 2007 nur noch 21,1 Prozent betrug, war die Unterrepräsentation der Jüngeren in der CSU 1990 genauso groß wie 2007 (PQ $1990=0,25,2007=0$,26) (vgl. Tabelle 8).

Die neue Alterstabelle ab 2008 mit vollständiger Vergleichbarkeit und feinerer Gliederung der Alterskategorien zeigt, dass eine Herabsetzung des Beitrittsalters unter 16 Jahre nur sehr wenige Mitglieder bringt. Auch der Anteil der Mitglieder bis 20 Jahre ist bei CDU, SPD, CSU und der Linken noch sehr gering und liegt deutlich unter dem der über 85-Jährigen. Der Anteil der jüngeren CDU-Mitglieder (bis 30 Jahre) stagnierte 2008 bis 2010 bei etwa sechs Prozent; bei der SPD ist er von 6,8 auf 7,5 Prozent gestiegen, in der CSU von 5,1 auf 4,7 Prozent gefallen. Die FDP zählt zwölf bis dreizehn Prozent Jüngere in ihrer Mitgliedschaft, bei der Linken stieg der Anteil von 6,4 auf 9,9 Prozent und bei den 
Grünen von 13,7 auf 16,8 Prozent. Die Linke weist 2010 mit 60 Jahren das höchste Durchschnittsalter der Parteimitglieder auf, gefolgt von CDU, SPD und CSU mit 58 Jahren. Die Grünen sind mit einem Durchschnittsalter von 46 Jahren die „jüngste“ Partei (vgl. Tabelle 9). Auch in ihren Reihen sind jedoch die 14- bis 20-Jährigen gegenüber der Bevölkerung sehr stark unterrepräsentiert, und selbst der Anteil der 21- bis 25-Jährigen an den Parteimitgliedern ist noch kleiner als in der Bevölkerung. Allerdings setzt sich die Unterrepräsentation bei CDU, SPD und Linken noch bis zur Altersgruppe der 46- bis 50-Jährigen fort. In der Mitgliedschaft von CDU, SPD, CSU und der Linken sind die Älteren (ab 61 Jahre) deutlich überrepräsentiert, in der FDP-Mitgliedschaft entsprechen sie in etwa dem Anteil unter den Beitrittsberechtigten, während sie in der Grünen-Mitgliedschaft deutlich unterrepräsentiert sind (vgl. Tabelle 10).

Für die CDU ist seit Mitte der 1990er Jahre ein kontinuierlicher Rückgang konfessionsgebundener Mitglieder festzustellen, der sich auch 2010 fortgesetzt hat. Dabei hat sich der Protestantenanteil deutlicher verringert als der Anteil der Katholiken. Bei der CSU schwanken die Werte etwas stärker, auch hier geht jedoch der Anteil an Katholiken und Protestanten zurück, wenn auch deutlich langsamer als bei der CDU. Allerdings waren Erstere von Anfang an in der Mitgliedschaft beider Parteien überrepräsentiert; und da der Katholikenanteil in der Bevölkerung noch etwas stärker abgenommen hat als in den Parteimitgliedschaften, hat sich die Überrepräsentation der Katholiken in der CDU und CSU in den letzten zwei Jahrzehnten nicht verringert, sondern sogar noch etwas erhöht. Der Katholikenanteil der CDU in Ostdeutschland ist zwar deutlich geringer als im Westen, setzt man ihn jedoch in Relation zur Bevölkerung, sind die Katholiken in der ostdeutschen CDUMitgliedschaft noch weit stärker überrepräsentiert als im Westen (vgl. Tabelle 11).

Die ab 2008 zur Verfügung stehenden Daten über Eintritte, Austritte und Todesfälle im Laufe des Jahres zeigen, dass bei CDU und SPD jeweils weniger als vier Prozent und bei der CSU weniger als drei Prozent neue Mitglieder hinzu kamen, wobei die Eintritte 2010 am geringsten waren. Deutlich größere Neumitgliederquoten hatten FDP, Grüne und Linke, wobei die FDP im Bundestagswahljahr 2009 mit fast 16 Prozent, die Grünen 2010 mit knapp 15 Prozent und die Linke 2008 mit fast 14 Prozent Neumitgliedern die höchsten Werte erreichten. Die Parteiaustritte bewegten sich in der Regel in der Größenordnung zwischen 2,5 und fünf Prozent, lediglich die FDP und die Linke mussten 2010 deutlich höhere Austrittsquoten von knapp acht beziehungsweise neun Prozent der Mitgliedschaft verkraften. Zudem verloren die Parteien - je nach Grad der Überalterung ihrer Mitgliedschaft - zwischen 0,2 und zwei Prozent ihrer Mitgliedschaft pro Jahr durch Todesfälle (vgl. Tabelle 12).

Der Frauenanteil bei den Eintritten liegt bei CDU, CSU und der Linken über dem Frauenanteil der Gesamtmitgliedschaft (vgl. Tabelle 13), und die Altersstruktur der Eintritte ist gegenüber der Gesamtmitgliedschaft wesentlich verjüngt (vgl. Tabelle 14 und 15). Besonders deutlich zeigt sich dies bei der SPD: Während zum Beispiel Ende 2009 weniger als vier Prozent der Gesamtmitgliedschaft bis 25 Jahre alt waren, betrug der Anteil dieser Altersgruppe bei den Neumitgliedern des Jahres 2010 fast 31 Prozent, bei den anderen Parteien nur 16 bis 21 Prozent. Wenn sich dieser Trend fortsetzt, könnte die SPD auf eine allmähliche leichte „Verjüngung“ hoffen. 


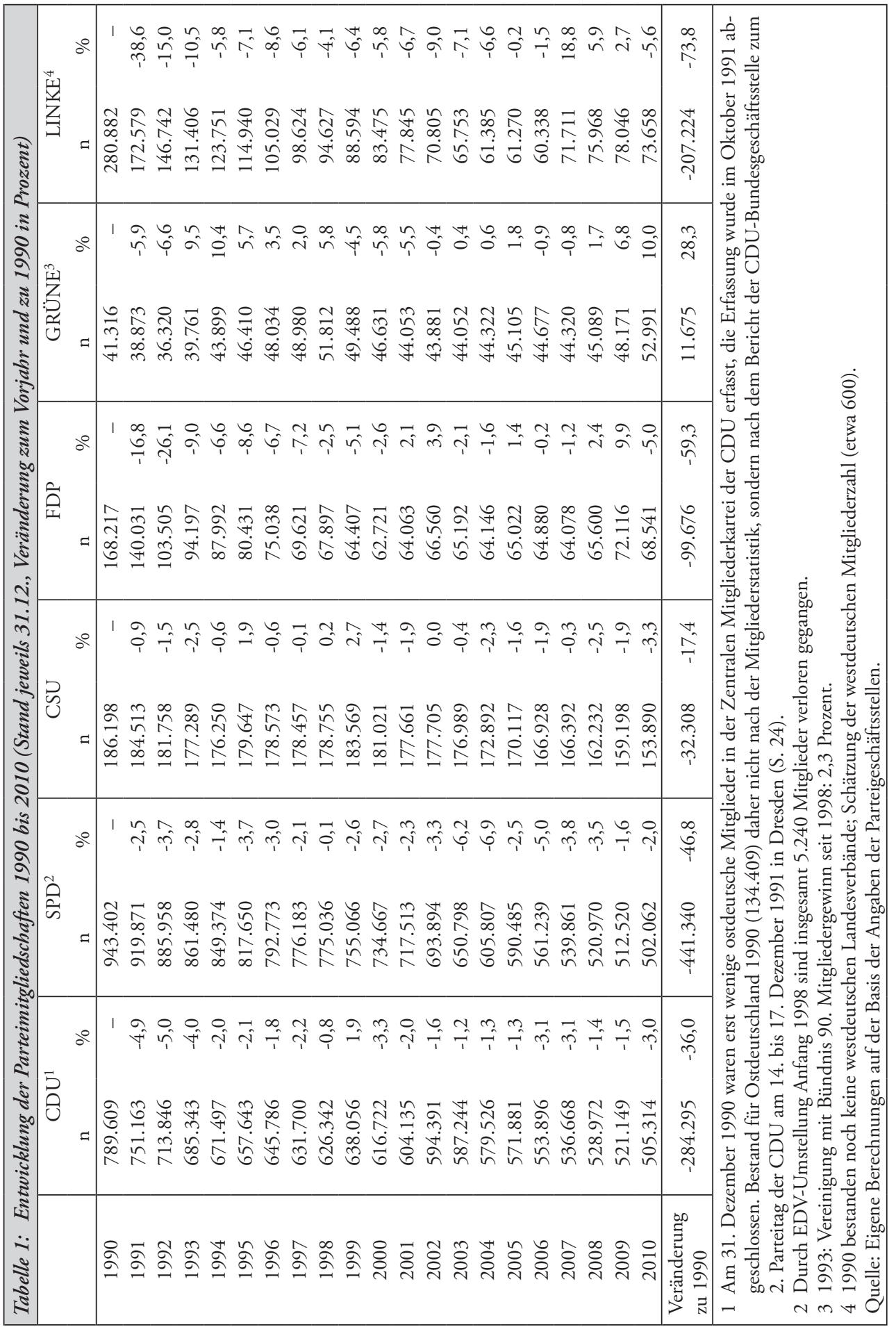




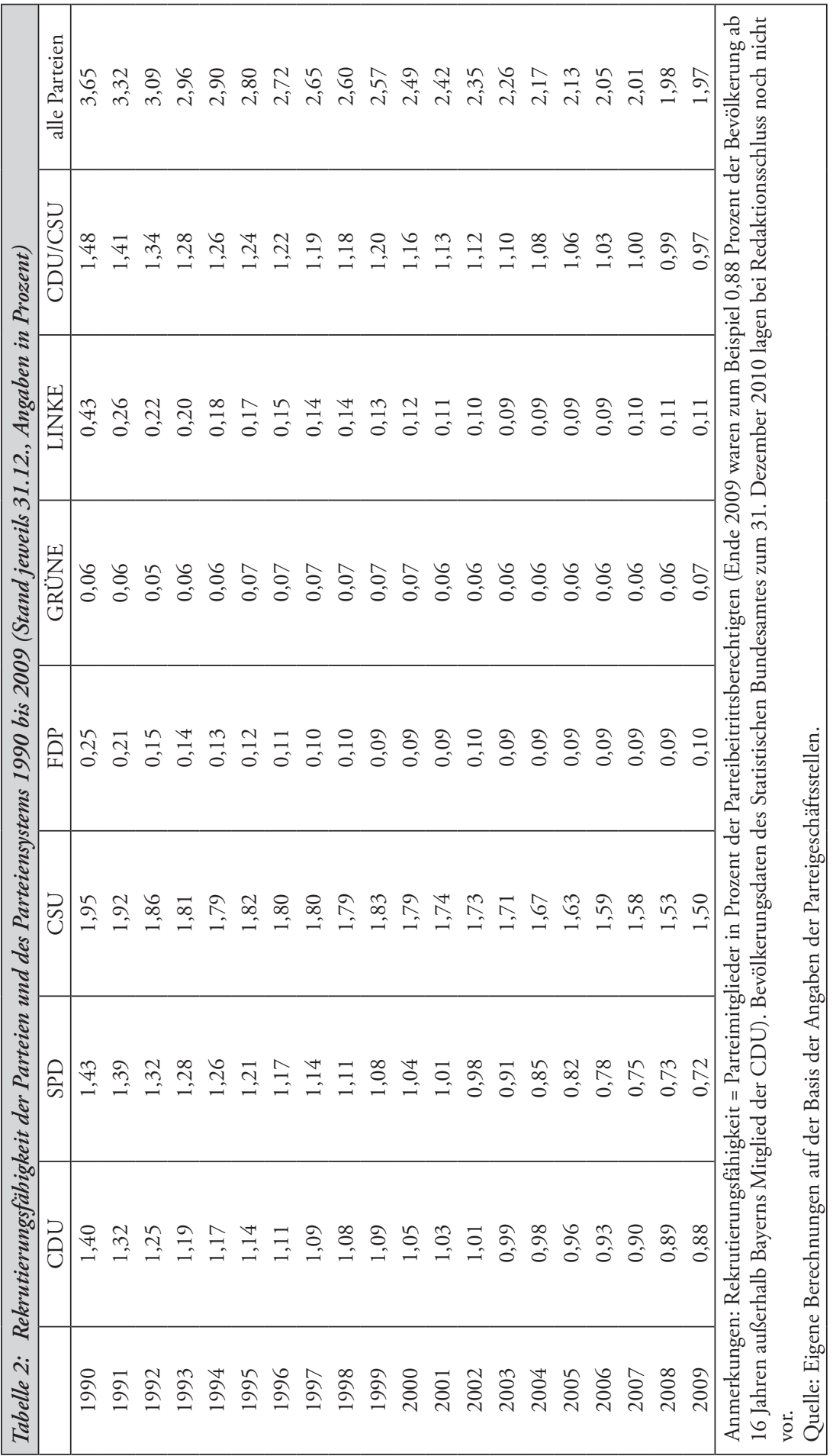




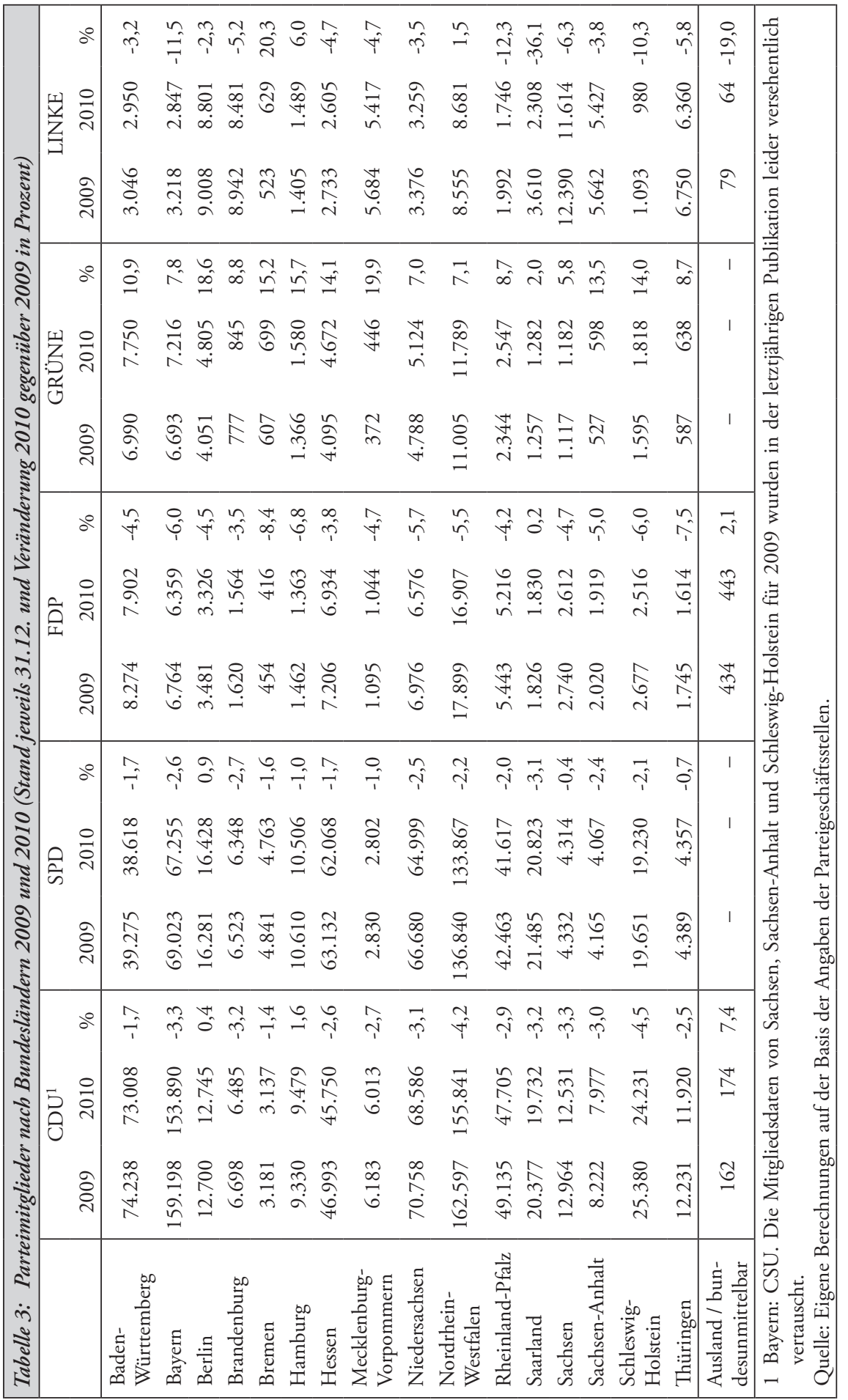




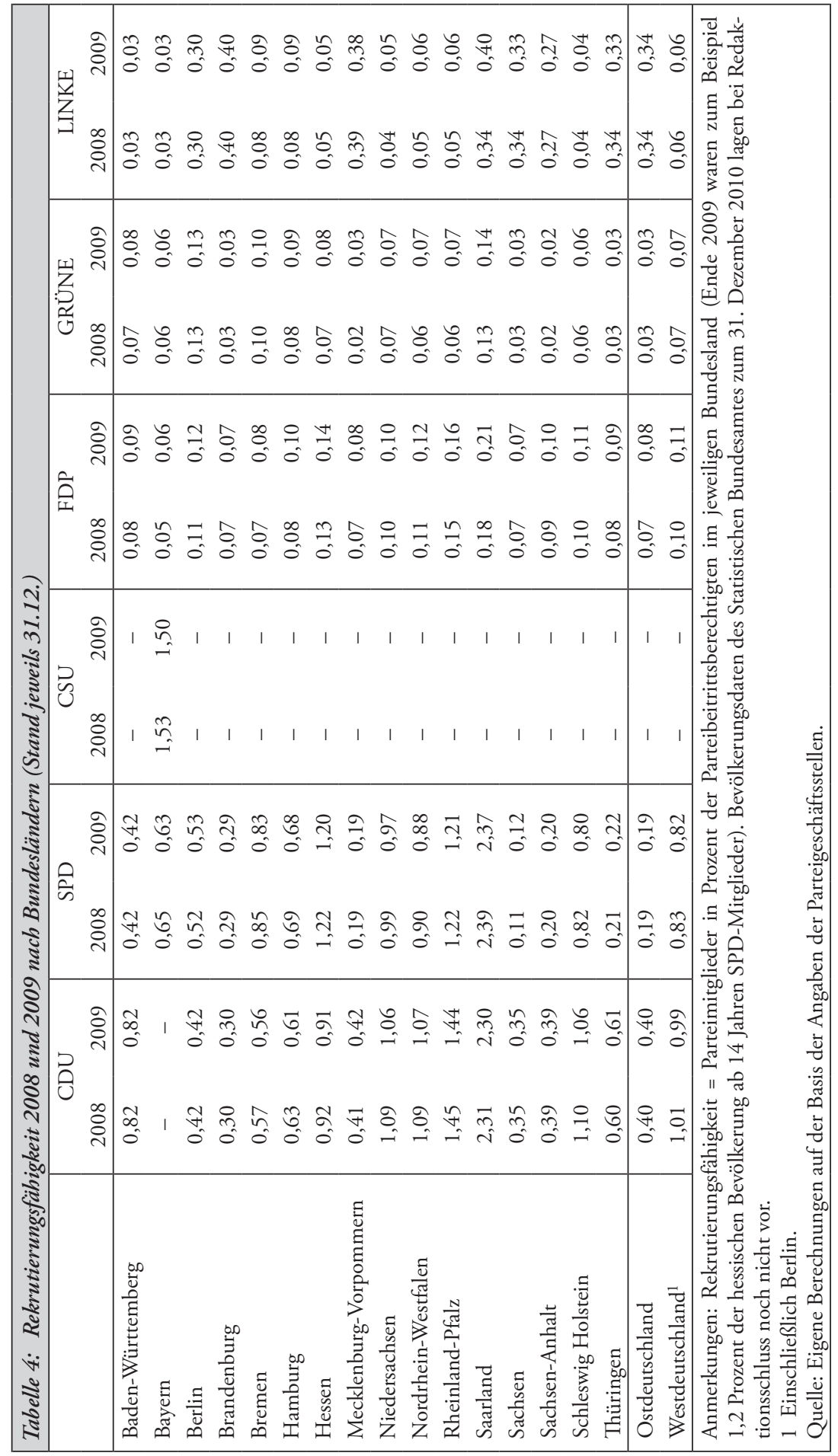




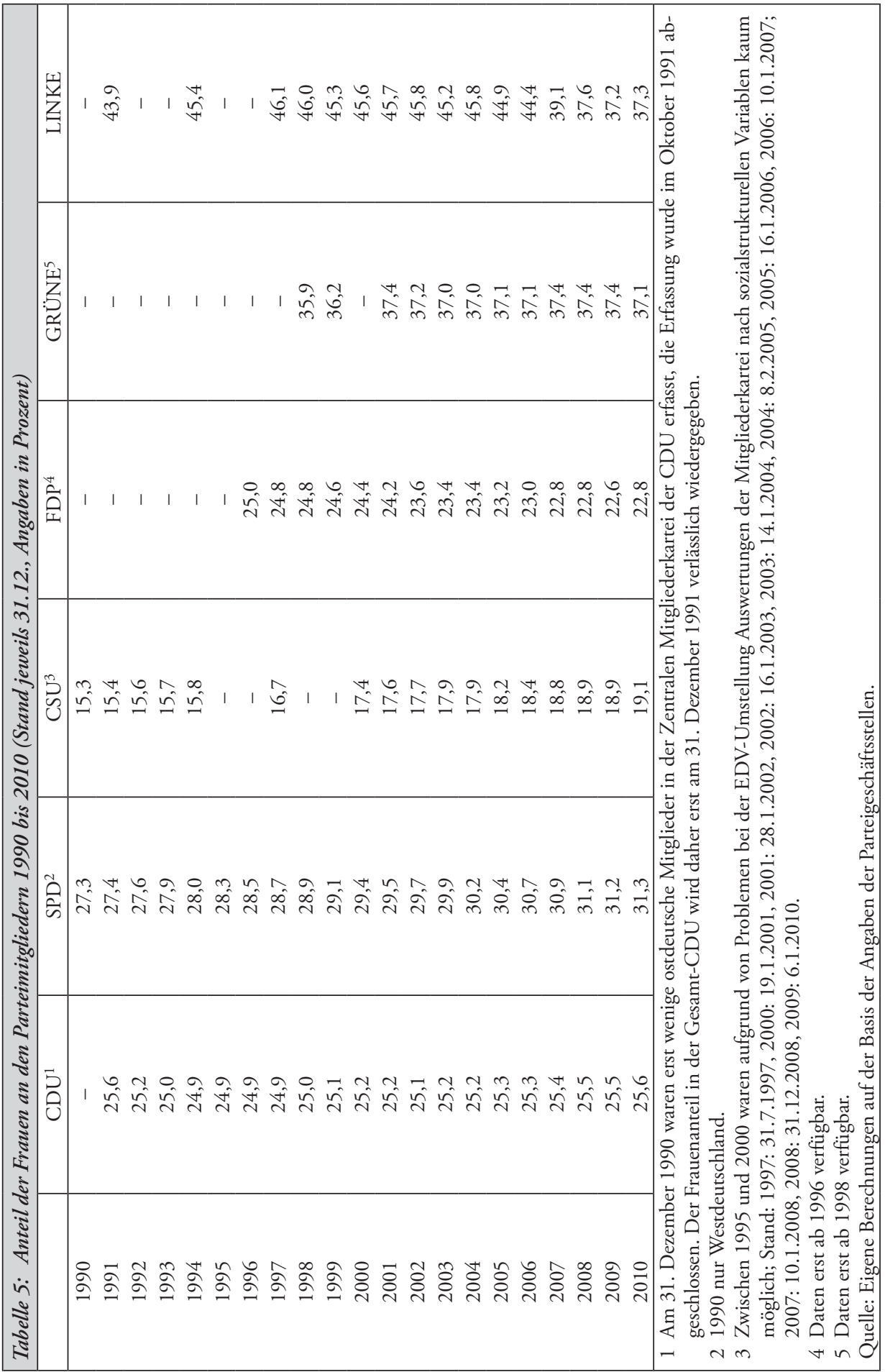




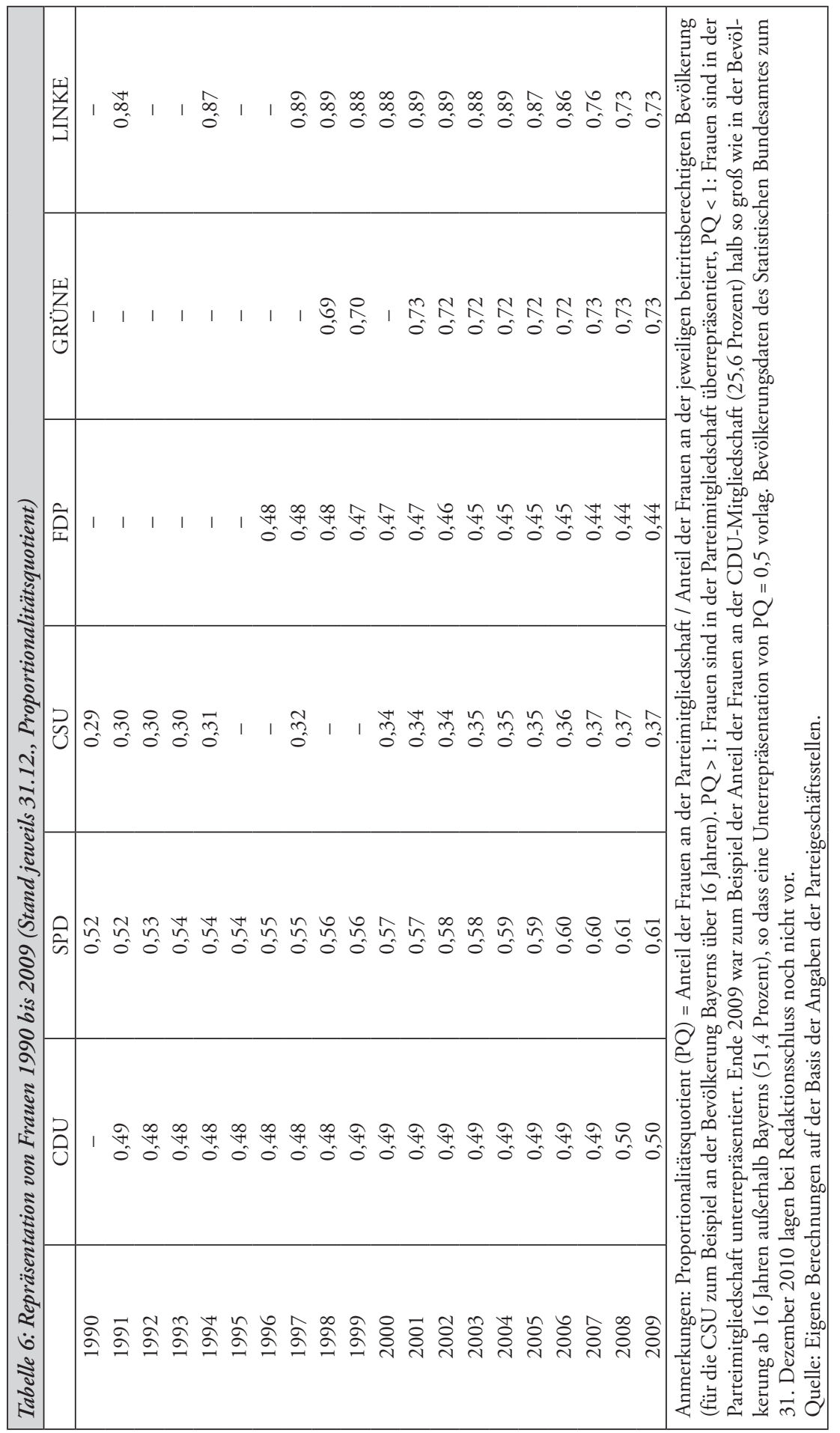




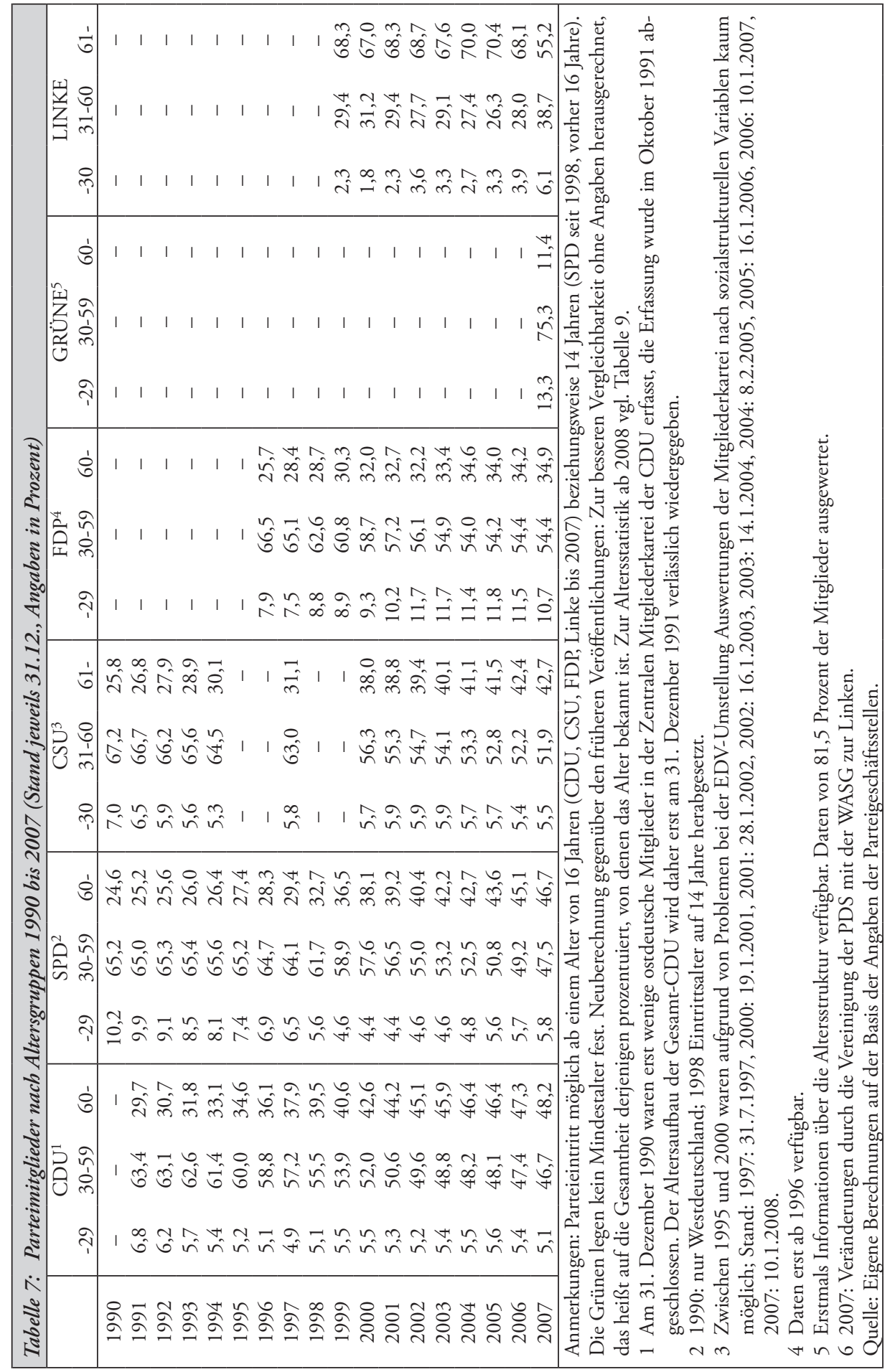




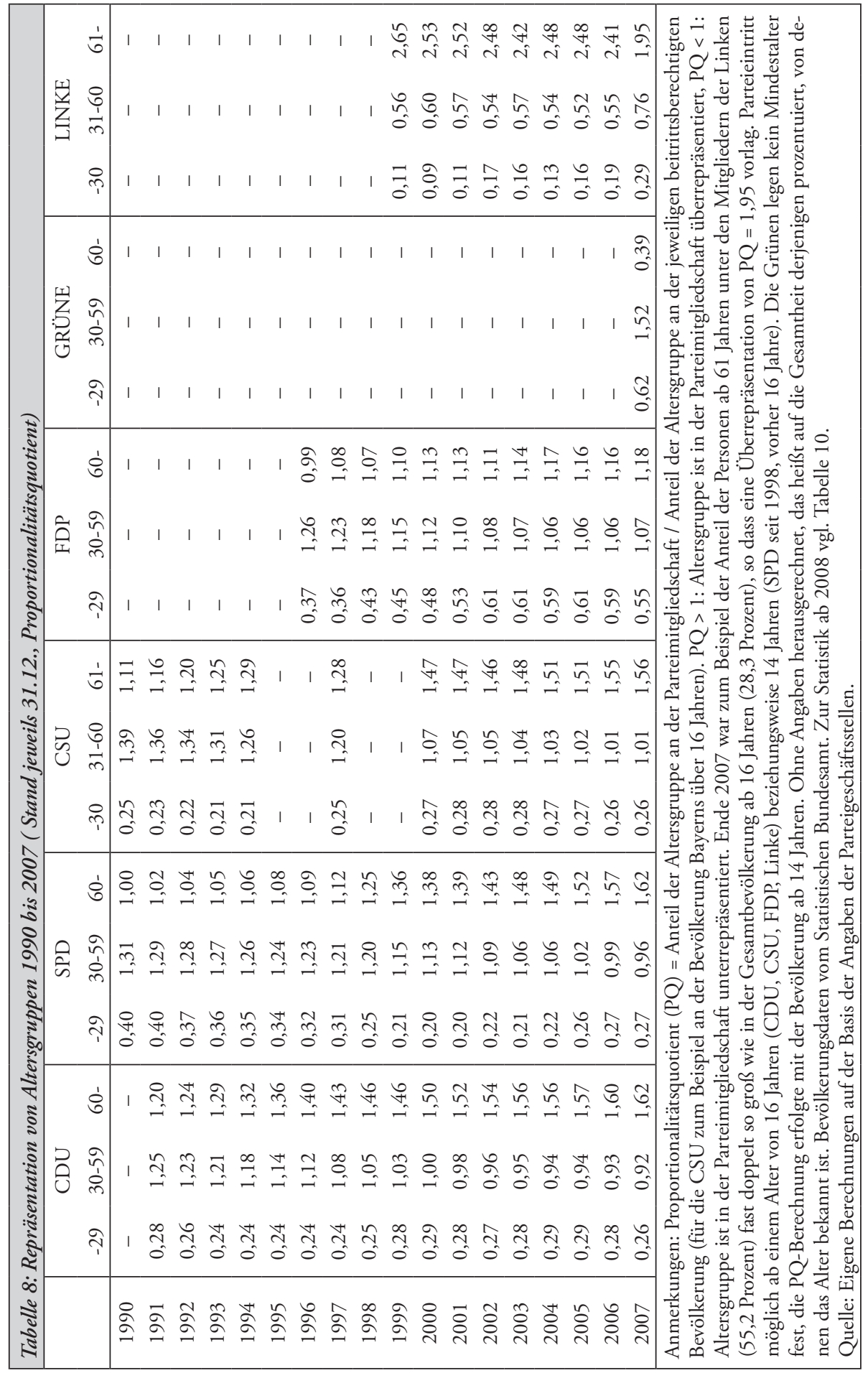




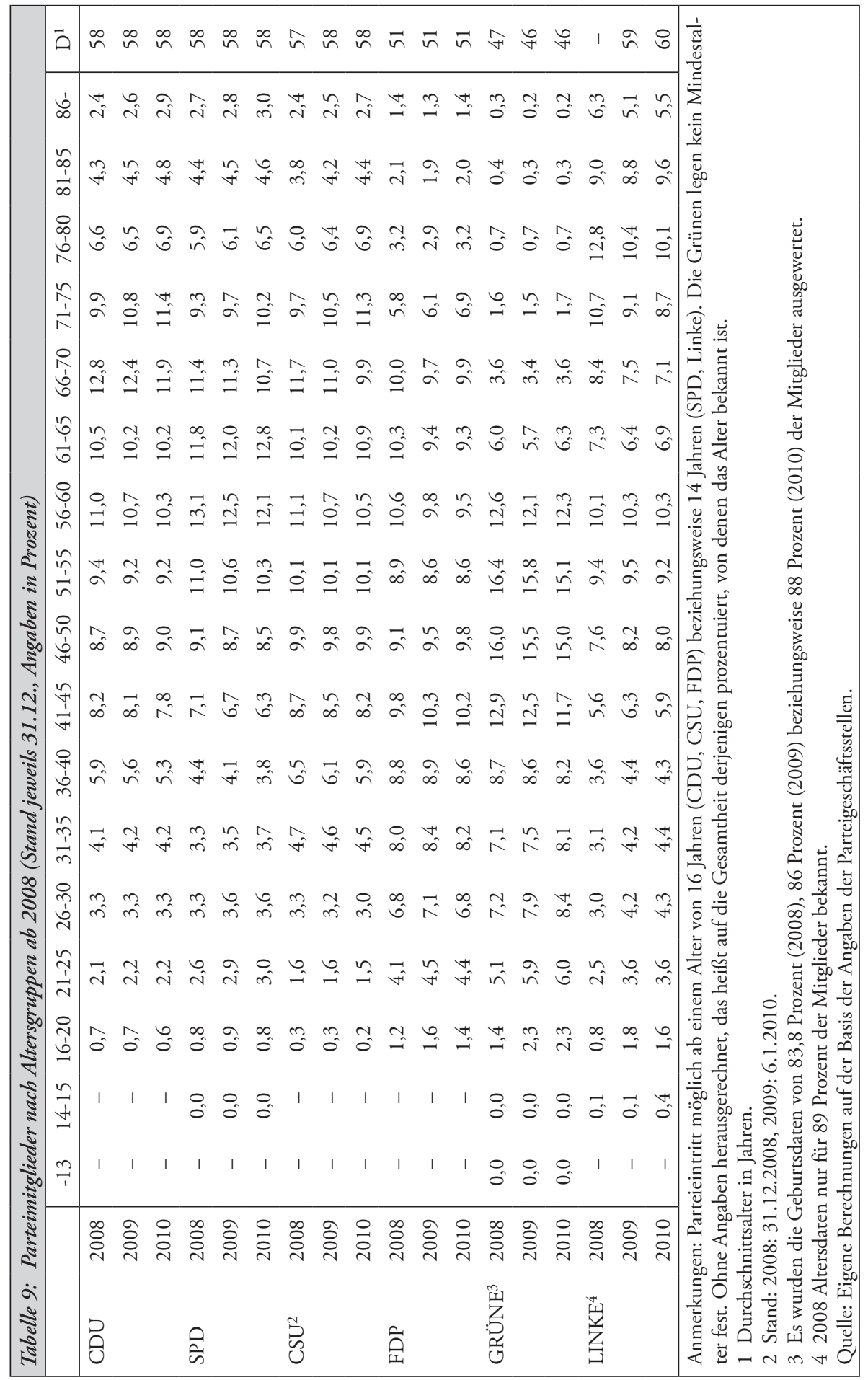




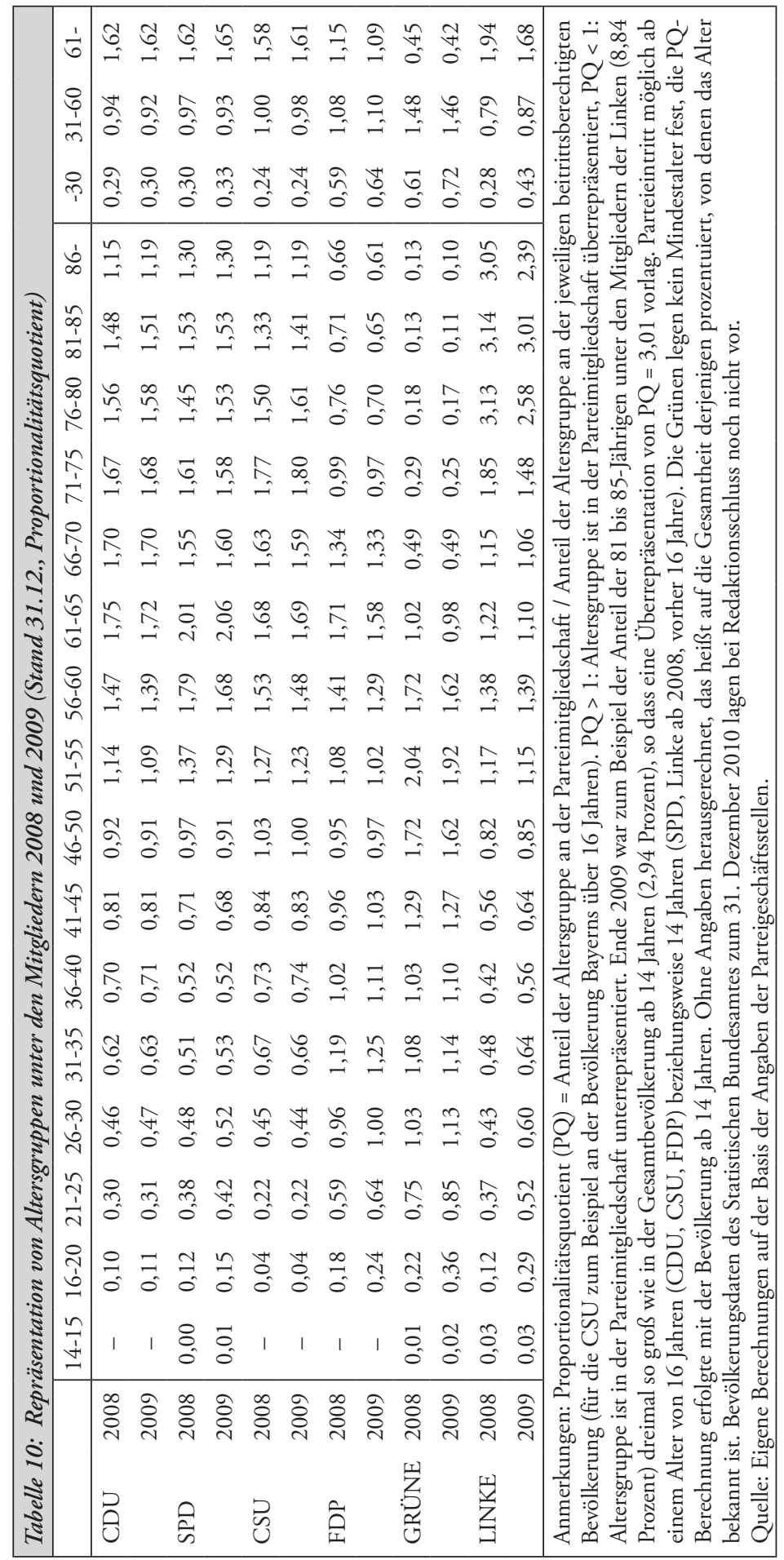




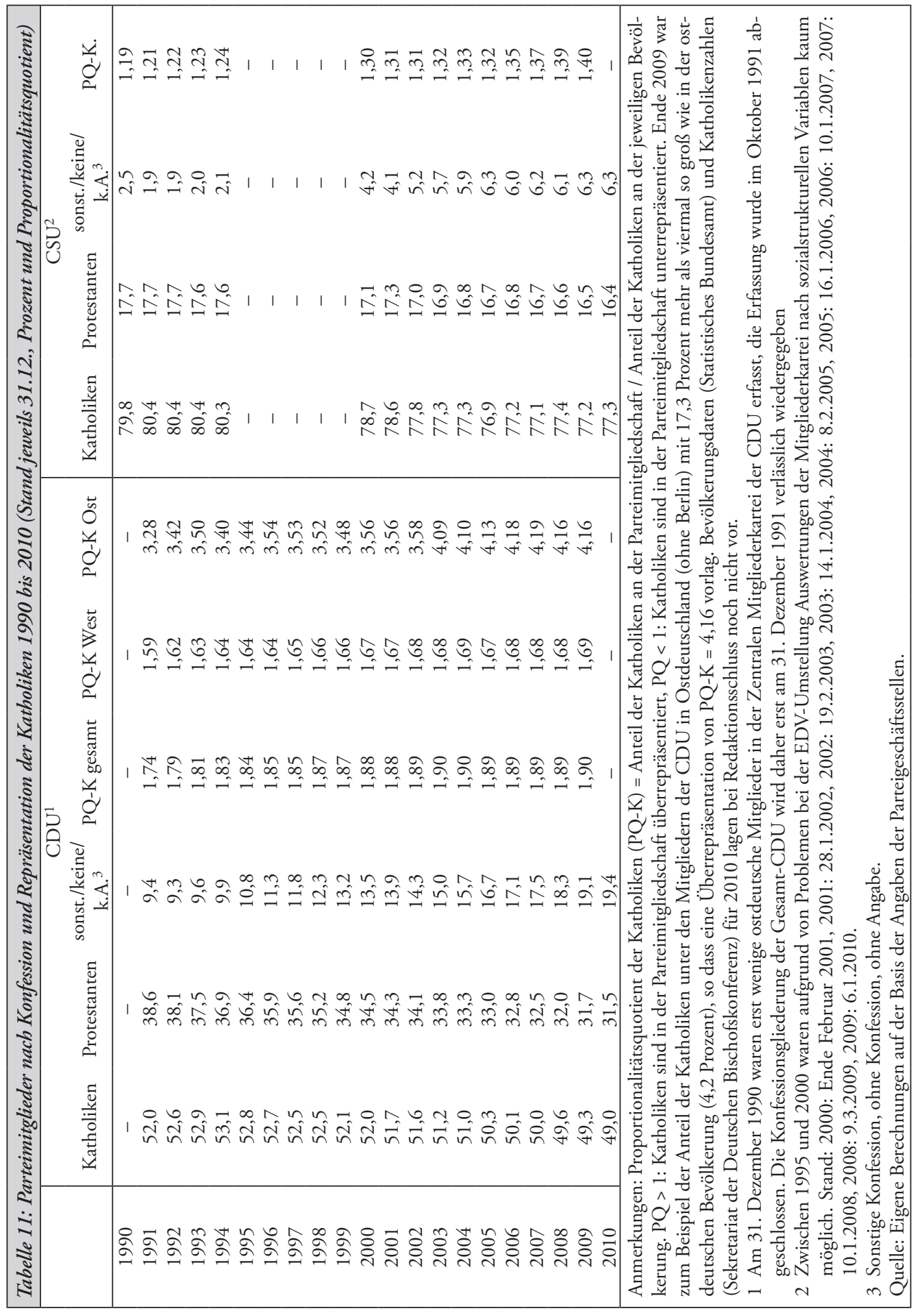




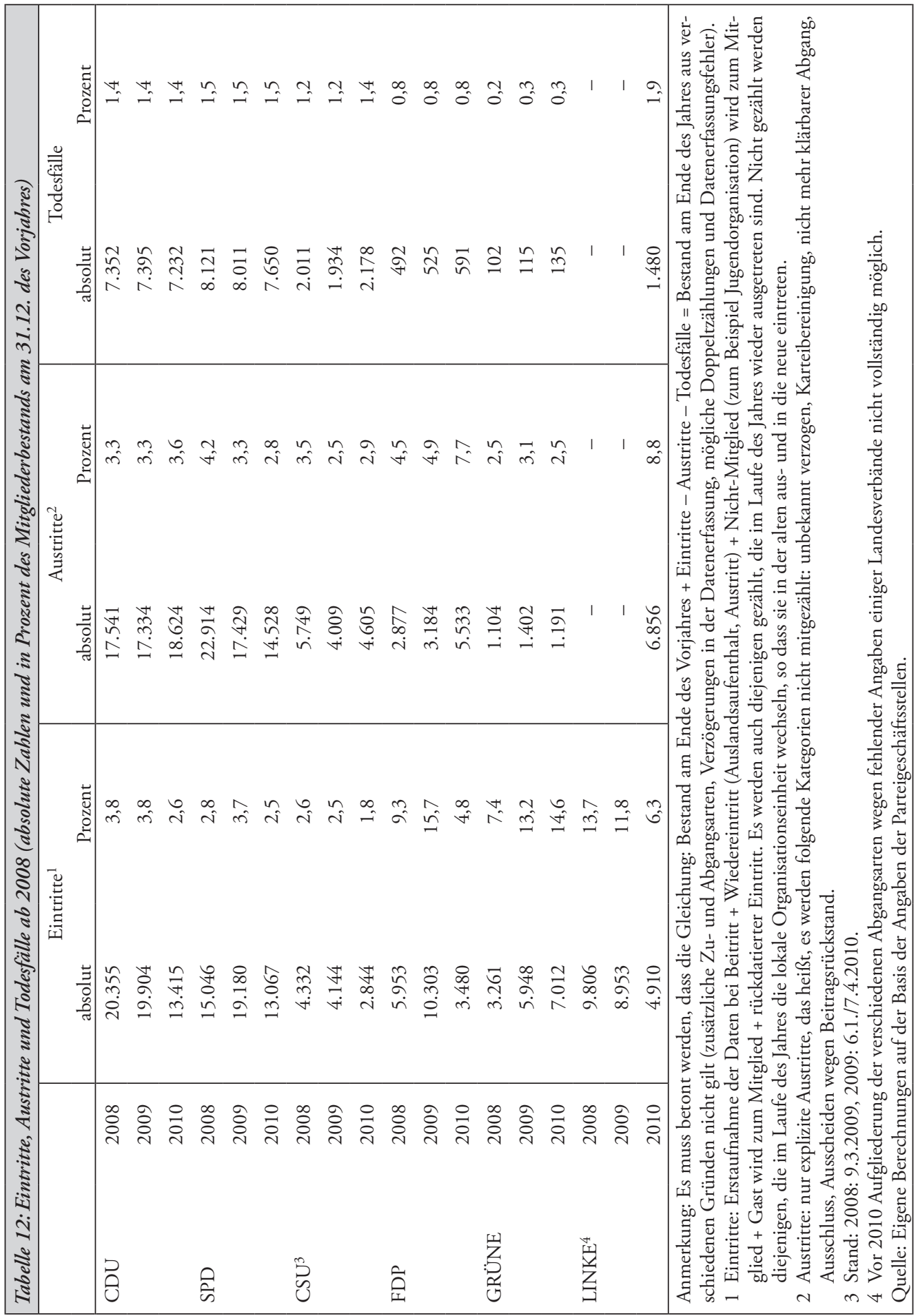




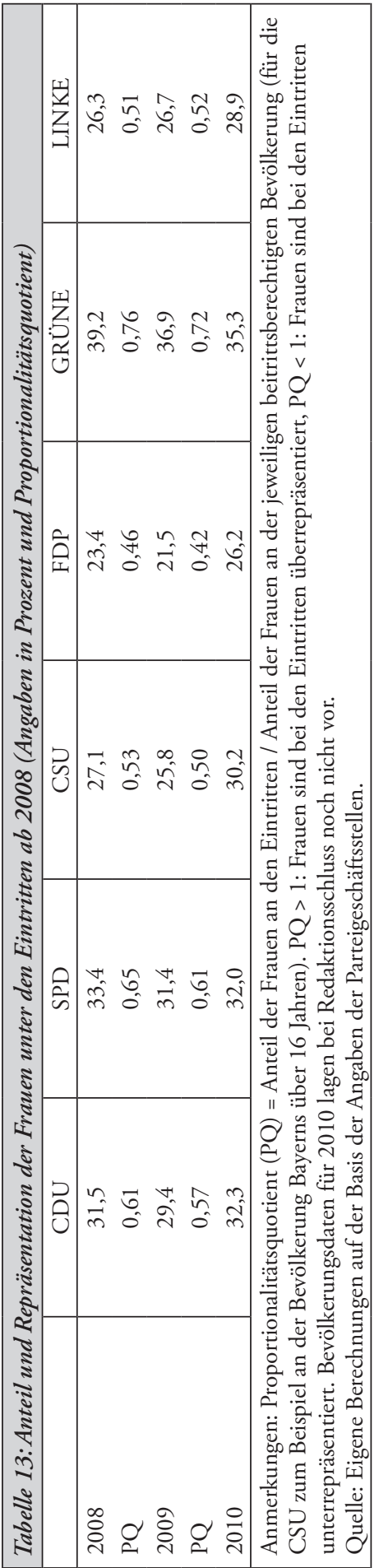




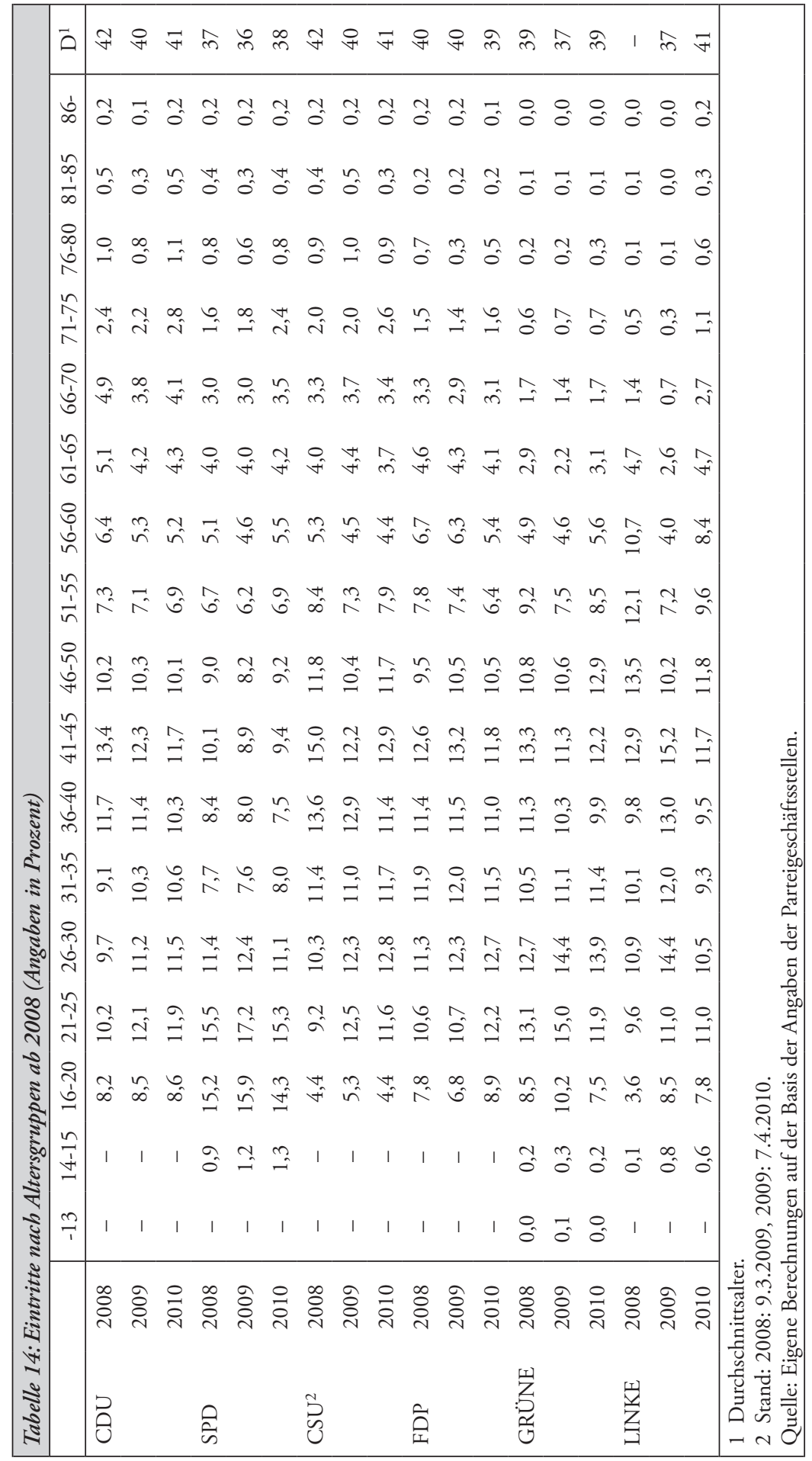




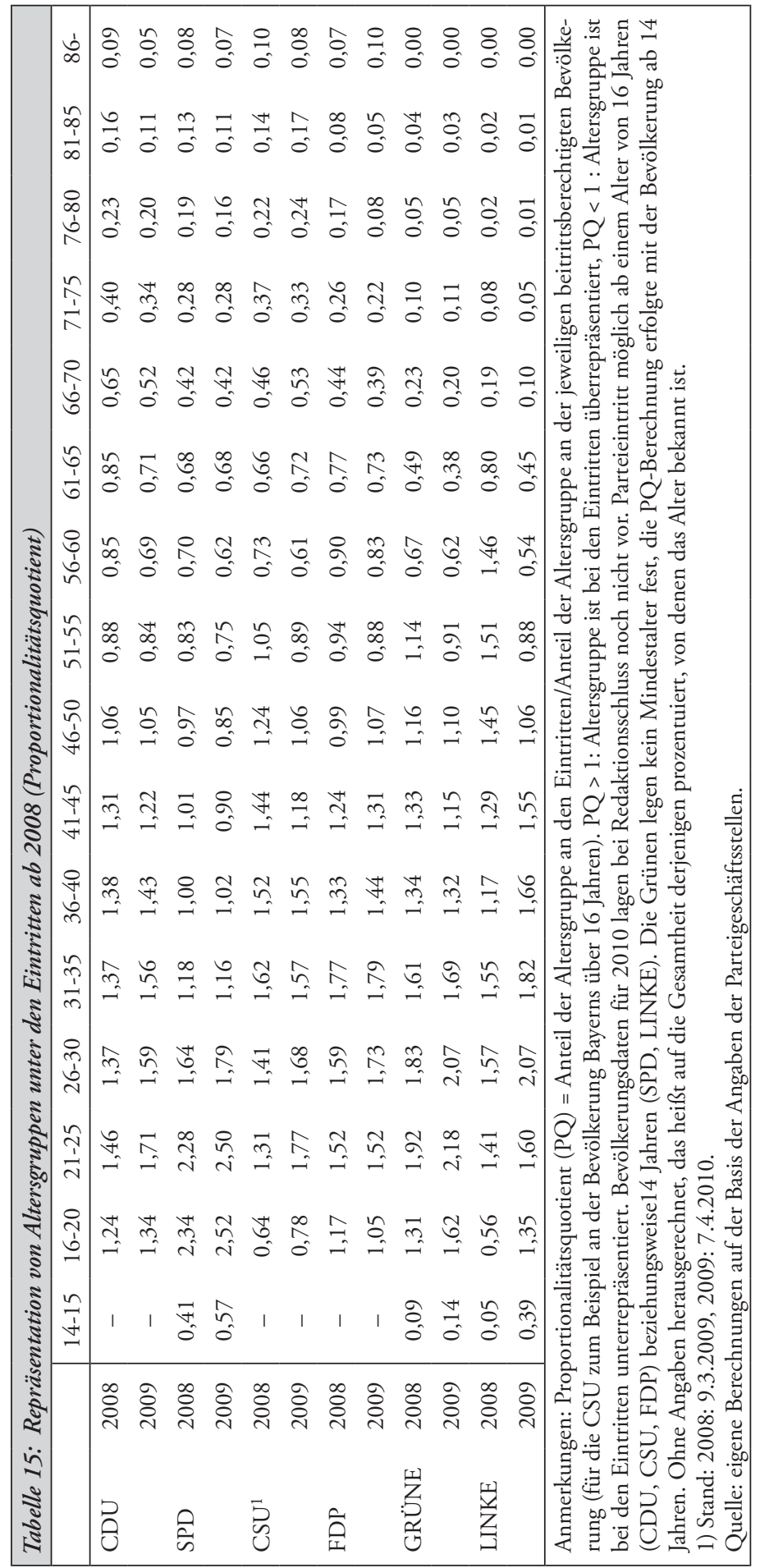

